\title{
Robust topological phase in proximitized core-shell nanowires coupled to multiple superconductors
}

\author{
Tudor D. Stanescu ${ }^{1}$, Anna Sitek ${ }^{2,3}$ and Andrei Manolescu ${ }^{* 3}$
}

\author{
Full Research Paper \\ Address: \\ ${ }^{1}$ Department of Physics and Astronomy, West Virginia University, \\ Morgantown, WV 26506, USA, ${ }^{2}$ Department of Theoretical Physics, \\ Faculty of Fundamental Problems of Technology, Wroclaw University \\ of Science and Technology, Wroclaw, 50-370, Poland and ${ }^{3}$ School of \\ Science and Engineering, Reykjavik University, Menntavegur 1, \\ IS-101 Reykjavik, Iceland \\ Email: \\ Andrei Manolescu* - manoles@ru.is \\ * Corresponding author \\ Keywords: \\ core-shell nanowires; Majorana states; multiple 1D chains; prismatic \\ geometry; topological superconducting phase
}

Beilstein J. Nanotechnol. 2018, 9, 1512-1526.

doi:10.3762/bjnano.9.142

Received: 03 December 2017

Accepted: 13 April 2018

Published: 22 May 2018

This article is part of the Thematic Series "Topological materials".

Guest Editor: J. J. Palacios

(C) 2018 Stanescu et al.; licensee Beilstein-Institut. License and terms: see end of document.

\begin{abstract}
We consider core-shell nanowires with prismatic geometry contacted with two or more superconductors in the presence of a magnetic field applied parallel to the wire. In this geometry, the lowest energy states are localized on the outer edges of the shell, which strongly inhibits the orbital effects of the longitudinal magnetic field that are detrimental to Majorana physics. Using a tight-binding model of coupled parallel chains, we calculate the topological phase diagram of the hybrid system in the presence of non-vanishing transverse potentials and finite relative phases between the parent superconductors. We show that having finite relative phases strongly enhances the stability of the induced topological superconductivity over a significant range of chemical potentials and reduces the value of the critical field associated with the topological quantum phase transition.
\end{abstract}

\section{Introduction}

The intense ongoing search for Majorana zero modes (MZMs) in solid states systems is motivated, in part, by the perspective of using them as a platform for fault-tolerant topological quantum computation [1-4]. Several practical realizations of "synthetic" topological superconductors that host zero-energy Majorana modes have been proposed in the past few years, the most promising involving semiconductor-superconductor hybrid systems [5-9]. The basic idea [10-13] is to proximity-couple a semiconductor nanowire with strong Rashba-type spin-orbit coupling (e.g., InSb or InAs) to a standard s-type superconductor (e.g., NbTiN or Al) in the presence of a longitudinal magnetic field. The system is predicted to host zero-energy Majorana modes localized at the two ends of the nanowire $[5,7,8]$. These zero-energy states combine equal proportions of electrons and holes and are created by second quantized operators satisfying the "Majorana condition" $\gamma^{\dagger}=\gamma$. The topological character of these modes endows them with robustness against perturbations that do not close the superconductor gap, e.g., 
weak interactions, wire bending, a certain amount of disorder, etc.

The most straightforward experimental signature of a Majorana mode is a zero-bias conductance peak that is produced in a charge transport measurement by tunneling electrons between the semiconductor wire and external electrodes attached to its ends [14-24]. These experiments have provided strong indications regarding the presence of Majorana bound states at the end of the wire, but no clear evidence of a phase transition to the topological phase, as revealed by the closing of the bulk quasiparticle gap [10-13], or evidence of correlated features at the opposite ends of the wire [25].

Ideally, the MZMs are hosted by a one-dimensional (1D) p-wave superconductor. However, the experimental realization and detection of these modes involve 3D nanowires [26]. The most common materials are InSb and InAs due to their large g-factor and strong SOC. The wires are grown by bottom-up methods and have usually a prismatic shape with a hexagonal cross section, as determined by the crystal structure [27]. The finite cross section of the wires used in the experiments may generate additional phenomena, which are not captured by ideal 1D models. In particular, the orbital effects of the magnetic field, which is oriented parallel to the nanowire, may reduce or even destroy the stability of the Majorana modes [28].

Proximitized core-shell nanowires are slightly more complex systems recently shown [29] to have interesting Majorana physics that is practically immune to orbital effects. With a conductive shell and an insulating core, such heterostructures become tubular conductors. The prismatic shape of the core-shell wires implies that the cross section of the shell can be seen as a polygonal ring. This is an interesting geometry because the corners of the polygon act like quantum wells where the states with the lowest energies are localized. Furthermore, a group of states with higher energies is localized on the sides of the polygon [30]. Although most of the core-shell nanowires have a hexagonal profile, square [31] or triangular [32-36] cross sections can also be obtained. The core diameter is typically between $50-500 \mathrm{~nm}$ and the shell thickness is between 1-20 nm. For all these geometries, the edge states corresponding to corner localization represent better approximations of the ideal 1D limit than the states hosted by a full wire. Remarkably, the energy separation between the corner states and the side states increases when the shell thickness is narrow compared to the radius of the wire, and when the corners are sharp. This means that the triangular shell would be the best choice for the realization of $1 \mathrm{D}$ edge channels. For example, with a shell thickens of $8-10 \mathrm{~nm}$ and a radius of $50 \mathrm{~nm}$ the energy separation between corner and side states can be be- tween 50-100 meV [29,37]. In this case the corner states are extremely robust to orbital effects of the magnetic field and the low-energy subspace is well separated from higher-energy states. Another interesting aspect of a prismatic shell is that it can host several Majorana states at each end of the wire. One can actually view the wire as a set of $n$ coupled chains, each having a pair of Majorana modes at its ends. On the one hand, this results in a rich phase diagram [29], which means that core-shell nanowires provide an interesting playground for studying topological quantum phase transitions. On the other hand, this richness is associated with rather fragile topological phases [29]. In practice, it would be extremely useful to have a knob enabling one to control the robustness of topological superconducting phase.

In this work we show that coupling a core-shell nanowire to two or more parent superconductors with non-vanishing relative phases enhances the stability of the topological phase and lowers the critical magnetic field associated with the (lowest field) topological quantum phase transition. In principle the phase difference between superconductors can be achieved either by applying an additional magnetic field, i.e., other than the longitudinal field needed for the Zeeman energy, or by driving a supercurrent through the superconductors. Hence, by controlling the relative phases of the parent superconductors coupled to the wire one can stabilize the topological superconducting phase that hosts the zero-energy Majorana modes and one can obtain an additional experimental knob for exploring a rich phase diagram and observing potentially interesting lowenergy physics.

The rest of this article is organized as follows. We first describe the coupled-chains tight binding model that we use in our numerical analysis. Then, using this simple model, we study the topological phase diagram of (infinite) core-shell wires with triangular and square cross section coupled to superconductors having the same superconducting phase. Next, we show that a finite phase difference can stabilize the topological phase in both triangular and square geometries. In addition, we show that the critical field associated with the (low-field) topological quantum phase transition can be made arbitrarily low. The implications of these findings for the stability of the Majorana modes emerging in finite wires is discussed in the subsequent section. Next, we corroborate our results for the topological phase diagram using an alternative "geometric" model. Finally, we summarize our findings and present our main conclusions.

\section{The Coupled-chains Tight-binding Model}

We start by formulating the effective thigh-binding model that describes the low-energy physics of a core-shell nanowire with 
$n$ edges. The model has already been introduced for triangular core-shell nanowires in [29] (Appendix), and also previously considered by other authors, in different forms, for ladder systems [38,39]. A "coarse-grained" shell is modeled by one chain associated with each vertex and one or more chains corresponding to each side, as shown in Figure 1. Note that the minimal model for a nanowire with $n$ edges consists of $2 n$ coupled chains ( $n$ for vertexes and $n$ for sides), but more detailed representations can be obtained by increasing the number of chains associated with the sides. A model that takes into account the details of the internal geometry of the wire [29] will be used later in the paper to corroborate the results obtained with this simple tight-binding model. In the numerical calculations we use minimal tight-binding models consisting of 6 (for triangular wires) or 8 (for square wires) parallel chains. Note that the odd chains, $\ell=1,3, \ldots$, correspond to the corners, while the even chains, $\ell=2,4, \ldots$, represent the sides.
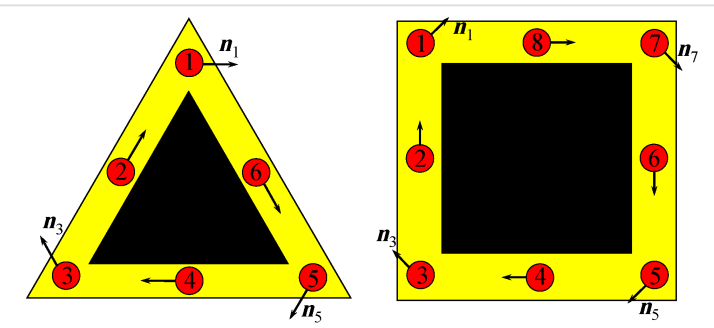

Figure 1: Schematic representation of the chain model for triangular (left) and square (right) core-shell nanowires. The shell (yellow) is coarse-grained so that the vertices and the sides are represented by 1D chains (red circles). The arrows indicate the direction of the effective spin-orbit field $\boldsymbol{n}_{\ell}$ associated with the (longitudinal) Rashba spinorbit coupling. In a minimal model each side is represented by one chain (left); a more detailed representation can be obtained by adding more chains associated with the sides (right).

Consider now $2 n 1 \mathrm{D}$ coupled chains proximity-coupled to one or more s-wave superconductors. The superconducting proximity effect is incorporated through the pairing potential $\Delta_{\ell}$, $1 \leq \ell \leq 2 n$ associated with each chain. Note that, in principle, the induced pairing potential may be chain-dependent. The lowenergy physics of the hybrid structure is described by the following Bogoliubov-de Gennes (BdG) Hamiltonian:

$$
\begin{aligned}
H= & -t \sum_{i, \ell, \sigma}\left(c_{i+1 \ell \sigma}^{\dagger} c_{i \ell \sigma}+h . c .\right)-t^{\prime} \sum_{i, \ell, \sigma}\left(c_{i \ell+1 \sigma}^{\dagger} c_{i \ell \sigma}+h . c .\right) \\
& +\sum_{i, \ell, \sigma}\left[V_{\text {eff }}(\ell)-\mu\right] c_{i \ell \sigma}^{\dagger} c_{i \ell \sigma}+\varepsilon_{0} \sum_{i, \sigma} \sum_{\ell}^{\text {even })} c_{i \ell \sigma}^{\dagger} c_{i \ell \sigma} \\
& +\frac{i}{2} \sum_{i, \ell}\left[\alpha c_{i+1 \ell}^{\dagger}\left(\hat{\boldsymbol{\sigma}} \cdot \boldsymbol{n}_{\ell}\right) c_{i \ell}+\alpha^{\prime} c_{i \ell+1}^{\dagger} \hat{\sigma}_{z} c_{i \ell}+h . c .\right] \\
& +\Gamma_{B} \sum_{i, \ell} c_{i \ell}^{\dagger} \hat{\sigma}_{z} c_{i \ell}+\sum_{i, \ell}\left(\Delta_{\ell} c_{i \ell \uparrow}^{\dagger} c_{i \ell \downarrow}^{\dagger}+\Delta_{\ell}^{*} c_{i \ell \downarrow} c_{i \ell \uparrow}\right),
\end{aligned}
$$

where $c_{i \ell \sigma}$ is the annihilation operator for an electron with spin projection $\sigma$ localized on the lattice site $i$ of the chain $\ell$ and $c_{i \ell}=\left(c_{i \ell \uparrow}, c_{i \ell \downarrow}\right)^{T}$ is the corresponding spinor operator. The first two terms in Equation 1 represent the nearest-neighbor hopping along the chains, with characteristic energy $t$, and the interchain coupling, with characteristic energy $t^{\prime}$. In the summations over the chain index $\ell$ we use the convention $2 n+1 \equiv 1$. The third term of the Hamiltonian (Equation 1) contains a chain-dependent effective potential $V_{\text {eff }}(\ell)$ that incorporates the presence of various external electrostatic fields (e.g., gate potentials) and the chemical potential $\mu$. Note that, in general, $V_{\text {eff }}(\ell)$ breaks the $n$-fold rotation symmetry of the original nanowire. The term proportional to $\varepsilon_{0}$ accounts for the fact that the side states have higher energies than the corner states and the parameter $\varepsilon_{0}>0$ controls the energy gap between the two types of states. The next term represents the Rashba type spin-orbit coupling (SOC), with longitudinal and transverse components proportional to $\alpha$ and $\alpha^{\prime}$, respectively. The underlying assumption is that the spin-orbit coupling is generated by an effective potential in the shell region due to the presence of the core [29]. The corresponding direction of the spin-orbit field $\boldsymbol{n}_{\ell}$ for electrons moving along the wire is shown in Figure 1. The next term in Equation 1, $\Gamma_{B}=g \mu_{b} B$, corresponds to the Zeeman spin splitting generated by an external magnetic field applied parallel to the wire (e.g., along the $z$-axis). The last term describes the proximity-induced pairing and takes into account the possibility that pairing potential $\Delta_{\ell}$ be chain-dependent. We assume that the vertex regions are covered by $n$ different superconductors separated by gaps over the side regions. The corresponding proximity-induced pairing potentials are

$$
\Delta_{\ell}= \begin{cases}0 & \text { if } \ell \text { is even } \\ \Delta \mathrm{e}^{i \phi_{\ell}} & \text { if } \ell \text { is odd }\end{cases}
$$

where $\phi_{\ell}$, the phase of the superconductor coupled to the vertex $\ell$, is an experimentally-controllable quantity. In the numerical calculations presented below we use the following values for the model parameters: $t=5.64 \mathrm{meV}, t^{\prime}=1.41 \mathrm{meV}$ (or $t^{\prime}=2.25 \mathrm{meV}$, when explicitly specified), $\alpha=2.0 \mathrm{meV}$, $\alpha^{\prime}=0.5 \mathrm{meV}, \varepsilon_{0}=15.0 \mathrm{meV}$, and $\Delta=0.3 \mathrm{meV}$.

To determine whether a given superconducting phase is topologically trivial or not, we calculate the $\mathbb{Z}_{2}$ topological index $\mathcal{M}$, i.e., the Majorana number [1],

$$
\mathcal{M}=\operatorname{sign}[\operatorname{Pf} B(0)] \operatorname{sign}[\operatorname{Pf} B(\pi)]
$$

The trivial and topological superconducting phases are characterized by $\mathcal{M}=+1$ and $\mathcal{M}=-1$, respectively. In Equation 3 
Pf[...] represents the Pfaffian [40], while the antisymmetric matrix $B(k)$ is the Fourier transform of the Hamiltonian (Equation 1) in the Majorana basis. The matrix $B(k)$ can be constructed using the particle-hole symmetry of the BdG Hamiltonian $[8,41]$,

$$
\mathcal{T} \mathcal{H}(k) \mathcal{T}^{-1}=\mathcal{H}(-k)
$$

where $\mathcal{H}(k)$ is the Fourier transform of the (single particle) Hamiltonian corresponding to Equation 1 and $\mathcal{T}=U_{t} K$ is the antiunitary time-reversal operator, with $U_{t}$ a unitary operator and $K$ the complex conjugation. Explicitly, we have

$$
B(\Lambda)=\mathcal{H}(\Lambda) U_{t}
$$

where $\Lambda=0, \pi / a$ are the time-reversal invariant points characterized by the property $\mathcal{H}(-\Lambda)=\mathcal{H}(\Lambda)$. The antisymmetry of $B(k)$ at the time-reversal invariant points, $B^{T}(\Lambda)=-B(\Lambda)$, is a direct consequence of Equation 4 and Equation 5. Considering that for typical parameter values the Pfaffian is always positive at the boundary of the Brillouin zone, $\operatorname{sign}[\operatorname{Pf} B(\pi)]=+1$, we conclude that the topological phase boundary is determined by a sign change of $\operatorname{Pf} B(0)$. Finally, using the general relation between the Pfaffian of a skew matrix $A$ and its determinant, $[\operatorname{Pf}(A)]^{2}=\operatorname{Det}(A)$, we have $\operatorname{Det} \mathcal{H}(0)=[\operatorname{Pf} B(0)]^{2}$. Note that $\operatorname{Det} \mathcal{H}(0)=0$ signals the presence of gapless states. Thus, the phase boundary, which corresponds to a sign change of the Pfaffian, is accompanied by the closing of the quasiparticle gap at $k=0$.

\section{Results and Discussion Nanowire coupled to superconductors with no relative phase difference}

The emergence of topological superconductivity and zeroenergy Majorana bound states in core-shell nanowires coupled to a single superconductor (i.e., in the absence of superconducting phase differences) was discussed in [29]. Here, we summarize the main results, as revealed by the simplified tightbinding model given by Equation 1. First, we consider a triangular system without a symmetry-breaking potential, $V_{\text {eff }}(\ell)=0$, and no superconducting phase difference, $\phi_{\ell}=0$. The corresponding topological phase diagram (as function of the chemical potential and the applied Zeeman field) is shown in panel (A) of Figure 2. The white regions correspond to $\mathcal{M}=+1$ (i.e., topologically trivial phases), while the orange areas represent topologically nontrivial phases with $\mathcal{M}=-1$. The effect of a symmetry-breaking potential is illustrated in panel (B) of Figure 2. While the topology of the phase diagram is the same, the phase boundaries are modified significantly with respect to panel (A). We note that this result was obtained by applying a rather modest symmetry breaking potential with values $V_{\text {eff }}=(0.67,0.17,-0.33,-0.33,-0.33,0.17) \mathrm{meV}$ on the six chains.

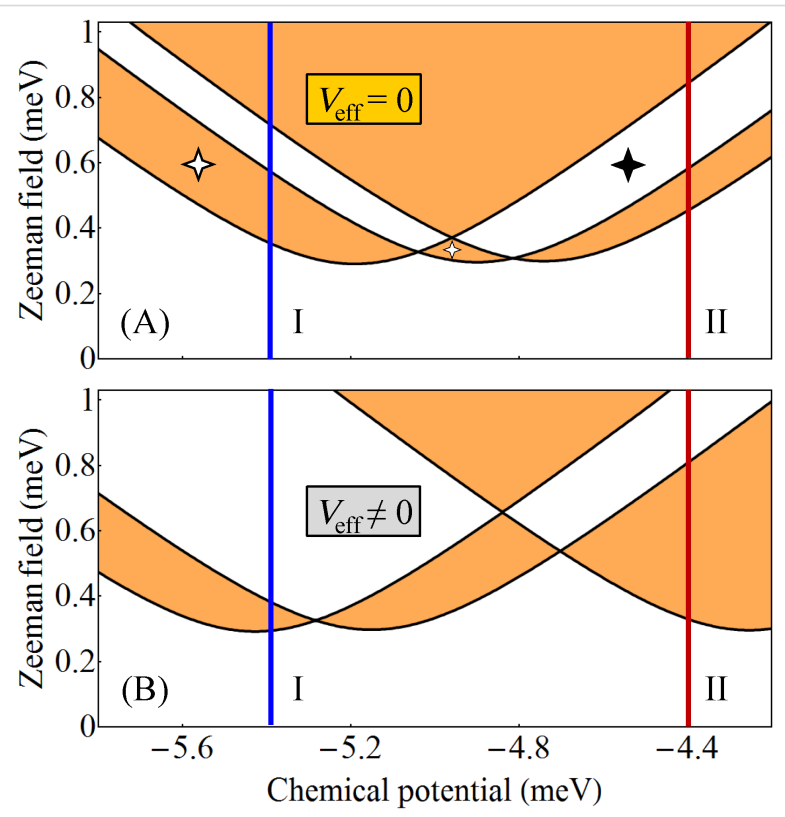

Figure 2: (A) Topological phase diagram for a triangular wire with $V_{\text {eff }}(\ell)=0$ and $\phi_{\ell}=0$. The white areas are topologically trivial and the orange regions are nontrivial. The 4-star symbols indicate gapless superconducting phases. (B) Topological phase diagram for a triangular wire with $V_{\text {eff }}(\ell) \neq 0$ and $\phi_{\ell}=0$. The values of the effective potential on the 6 chains are $(0.67,0.17,-0.33,-0.33,-0.33,0.17)$ $\mathrm{meV}$. The evolution of the (minimum) quasiparticle gap along the cuts I (blue lines) corresponding to $\mu=-5.4 \mathrm{meV}$ and II (red lines) corresponding to $\mu=-4.4 \mathrm{meV}$ are shown in Figure 3 and Figure 4, respectively. See also [29].

To get further insight into the nature of the phases shown in Figure 2, we calculate the minimum quasiparticle energy $E_{\min }\left(\mu, \Gamma_{B}\right)$ along the constant chemical potential cuts I (blue) and II (dark red) marked on the phase diagrams. This energy (which corresponds to the minimum quasiparticle gap) is defined as

$$
E_{\min }\left(\mu, \Gamma_{B}\right)= \pm \min _{n, k}\left|E_{n}(k)\right|
$$

where $E_{n}(k)$ are the eigenvalues of the BdG Hamiltonian from Equation 1. The dependence of $E_{\min }$ on the Zeeman field for $\mu=-5.4 \mathrm{meV}$ (i.e., the blue cuts I in Figure 2) is shown in Figure 3, while the evolution of the minimum gap along the cuts II (dark red) corresponding to $\mu=-4.4 \mathrm{meV}$ is shown in Figure 4.

At zero Zeeman field, $\Gamma_{B}=0$, the system is in a trivial superconducting phase characterized by a quasiparticle gap 


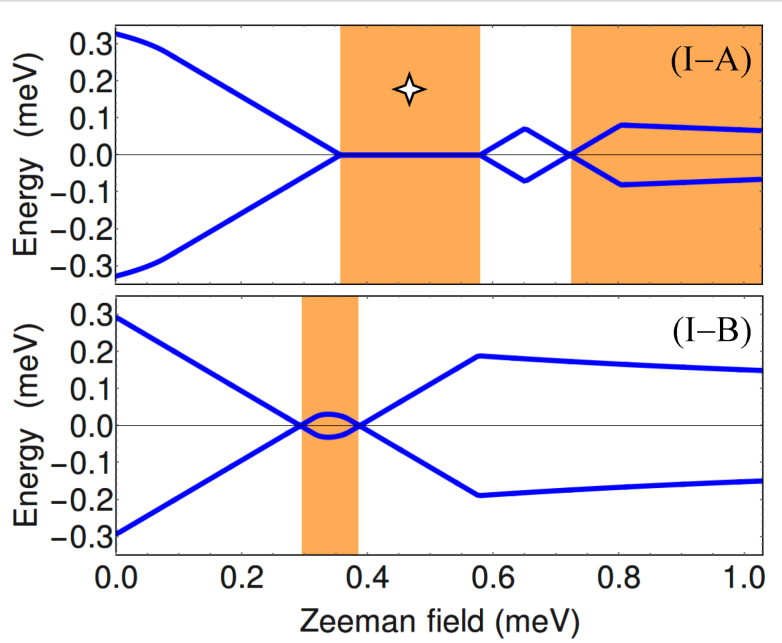

Figure 3: Dependence of the minimum quasiparticle gap on the Zeeman field along the blue cuts (I) corresponding to $\mu=-5.4 \mathrm{meV}$ in Figure Figure 2. Top: $V_{\text {eff }}(\ell)=0$, see Figure $2 A$. Bottom: $V_{\text {eff }}(\ell) \neq 0$, see Figure $2 \mathrm{~B}$. The white/orange regions correspond to the trivial/ nontrivial phases shown in Figure 2. Note the gapless superconducting phase marked be the 4-star symbol (top panel). See also [29].

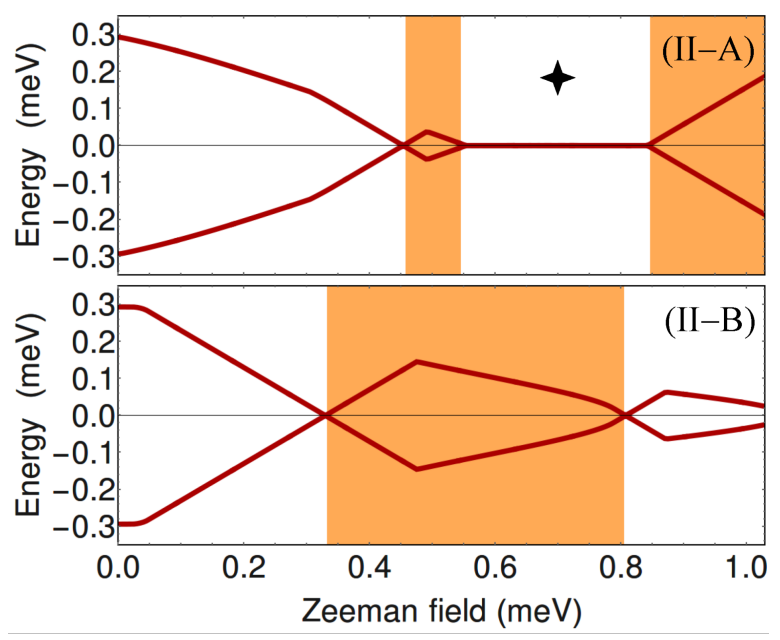

Figure 4: Dependence of the minimum quasiparticle gap on the Zeeman field along the dark red cuts (II) corresponding to $\mu=-4.4 \mathrm{meV}$ in Figure 2. Top: $V_{\text {eff }}(\ell)=0$, see Figure $2 A$. Bottom: $V_{\text {eff }}(\ell) \neq 0$, see Figure $2 B$. The white/orange regions correspond to the trivial/nontrivial phases shown in Figure 2. Note the gapless superconducting phase marked be the 4-star symbol (top panel). See also [29].

$\Delta=0.3 \mathrm{meV}$ (see Figure 3 and Figure 4 ) given by the value of the induced pairing potential. With increasing $\Gamma_{B}$, the quasiparticle gap reduces and eventually closes at a certain critical Zeeman energy. In the absence of a symmetry breaking potential, the system with $\mu=-5.4 \mathrm{meV}$ (see cut (I-A) in Figure 2) remains gapless throughout the first (i.e., low-field) orange region, which means that the system becomes a gapless superconductor. Another gapless superconducting phase corresponds to the intermediate white region in panel (II-A) of Figure 4, i.e., for Zeeman fields between approximately $0.55 \mathrm{meV}$ and $0.85 \mathrm{meV}$. These gapless phases are marked by a 4-star symbol in the phase diagram (see Figure 2A) and in Figure 3(I-A) and Figure 4(II-A). We note that inside the gapless superconducting phases the gap closes at $k \neq 0$. Of course, at the phase boundaries the gap always closes at $k=0$. Furthermore, by increasing the Zeeman energy above $0.7 \mathrm{meV}$ in panel (I-A) of Figure 3 or above $0.85 \mathrm{meV}$ in panel (II-A) of Figure 4, the system evolves into topological phase with a finite gap.

Upon breaking the three-fold rotation symmetry of the original triangular wire, the gapless superconducting phases become gapped. Also notice in panel (II-B) that the low-field topological phase corresponding to $\mu=-4.4 \mathrm{meV}$ is now characterized by a sizable quasiparticle gap, indicating a regime which may be more favorable for robust zero-energy Majorana modes. We note that the robust low-field topological phase in panel (II-B) corresponds to a single pair of Majorana modes (i.e., one MZM at each end of the wire) hosted by chain 1 (with the highest

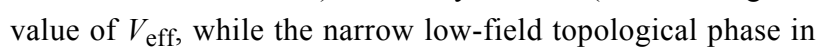
panel (I-B) corresponds to a pair of Majorana modes shared by chains 2 and 3 (the chains with the lowest value of the potential). Note that the expression "hosted by chain 1" (or chains 2 and 3) actually means that most of spectral weight associated with the Majorana wave function is localized on the corresponding chain(s) (also see below, Figure 11 and Figure 13). The wide trivial region above $\Gamma_{B} \approx 0.4 \mathrm{meV}$ in panel (I-B) corresponds to a finite system with two pairs of Majorana bound states (on chains 2 and 3). We also note that the low-field phase boundaries converge to a single boundary in the limit of isolated chains, i.e., when the inter-chain hopping energy is much smaller than the hopping along the chains, $t^{\prime} / t \rightarrow 0$. In this case three Majorana pairs would form independently at the ends of each chain, and coexist at zero energy, without "talking" to each other. Physically, the limit $t^{\prime} / t \rightarrow 0$ corresponds an infinitely-thin shell. For finite values of $t^{\prime} / t$ (corresponding to finite shell thicknesses), the coupling between chains lifts the degeneracy, such that at most one Majorana state can have zero energy, while the other two will acquire finite energy.

The existence of gapless superconducting phases in systems with rotation symmetry is generic, i.e., it holds for $n>3$. We emphasize that gapless phases cannot host stable Majorana modes and, therefore, they are not suitable for studying Majorana physics. Applying a symmetry-braking potential $V_{\text {eff }}(\ell) \neq 0$ opens a finite gap throughout the entire phase diagram, except, of course, the phase boundaries, where the quasiparticle gap vanishes at $k=0$. To better illustrate this point, we calculate the topological phase diagram for a square wire with $V_{\text {eff }}(\ell) \neq 0$ and the minimum gap along a representative cut through the phase diagram. The results are shown in 
Figure 5. Note that all topologically trivial and nontrivial phases are gapped. However, the gaps are rather small indicating the fact that topological superconductivity (and the corresponding Majorana modes) are not very robust.
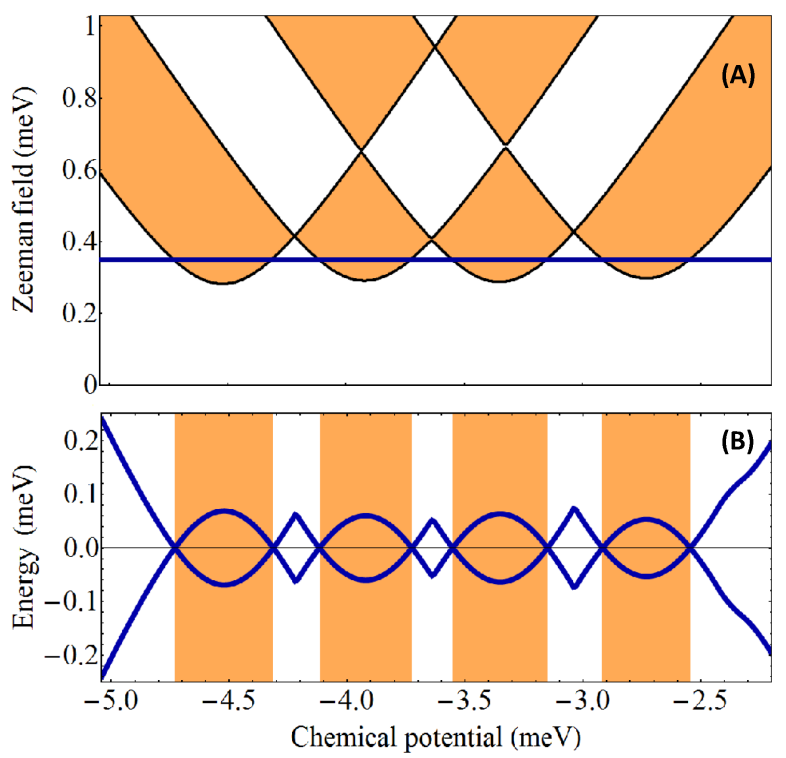

Figure 5: (A) Topological phase diagram for a square wire with $V_{\text {eff }}(\ell) \neq 0$ and $\phi_{\ell}=0$. The white areas are topologically trivial and the orange regions are nontrivial. The values of the effective potential on the 8 chains are $(0.5,0,-0.5,-0.5,-0.5,0,0.5,0.5) \mathrm{meV}$ and the inter-chain hopping is $t^{\prime}=2.25 \mathrm{meV}$. (B) Evolution of the minimum quasiparticle gap along the horizontal cut $\Gamma=0.35 \mathrm{meV}$ shown in the top panel.

An important difference between the phase diagram shown in Figure 5 and that in Figure 2 is that for the square wire we have used a larger value of the inter-chain hopping, $t^{\prime}=2.25 \mathrm{meV}$. Enhancing the coupling between chains widens the low-field topological regions (which would practically vanish in the limit $t^{\prime} / t \rightarrow 0$ ). Finally, we emphasize that although a finite system with parameters corresponding to a topologically nontrivial phase will support one pair of MZMs (i.e., one Majorana mode at each end of the wire), generically each Majorana mode is hosted by multiple chains (rather than a single chain). For example, in a configuration corresponding to Figure 5, the lowfield topological phases with $\mu<3.7 \mathrm{meV}$ can support MZMs hosted by chains 3 and 5 (with minimum values of $V_{\text {eff }}(\ell)$ ), while for $\mu>3.7 \mathrm{meV}$ the MZMs are hosted by chains 1 and 7 (corresponding to the maximum values of $V_{\mathrm{eff}}(\ell)$ ).

\section{Wires coupled with multiple superconductors: the stabilizing role of the phase difference}

A critical question that we want to investigate concerns the effect of a nonzero superconducting phase difference in a wire coupled to multiple parent superconductors. A non-zero phase difference was shown to stabilize the topological phase in a Josephson junction across a 2D electron gas with Rashba spinorbit coupling and in-plane magnetic field [42] and in a topological insulator nanoribbon coupled with two superconductors [43]. Here, for concreteness, we consider a triangular core-shell nanowire modeled by six chains, as described above, which are coupled to three separate superconductors that induce pairing potentials characterized by $\phi_{1}=0, \phi_{3}=\pi / 2$, and $\phi_{5}=-\pi / 2$. The other parameters are the same as in Figure 2B, i.e., the case $V_{\text {eff }} \neq 0$ discussed above. The corresponding phase diagram is shown in Figure 6. Remarkably, the "crossing points" that characterize the phase diagram in Figure 2 disappear and, upon increasing the Zeeman field, we have an alternance of trivial and nontrivial phases for all values of the chemical potential. More importantly, the low-field topological phase becomes stable for a wide range of chemical potentials, i.e., it is characterized by a
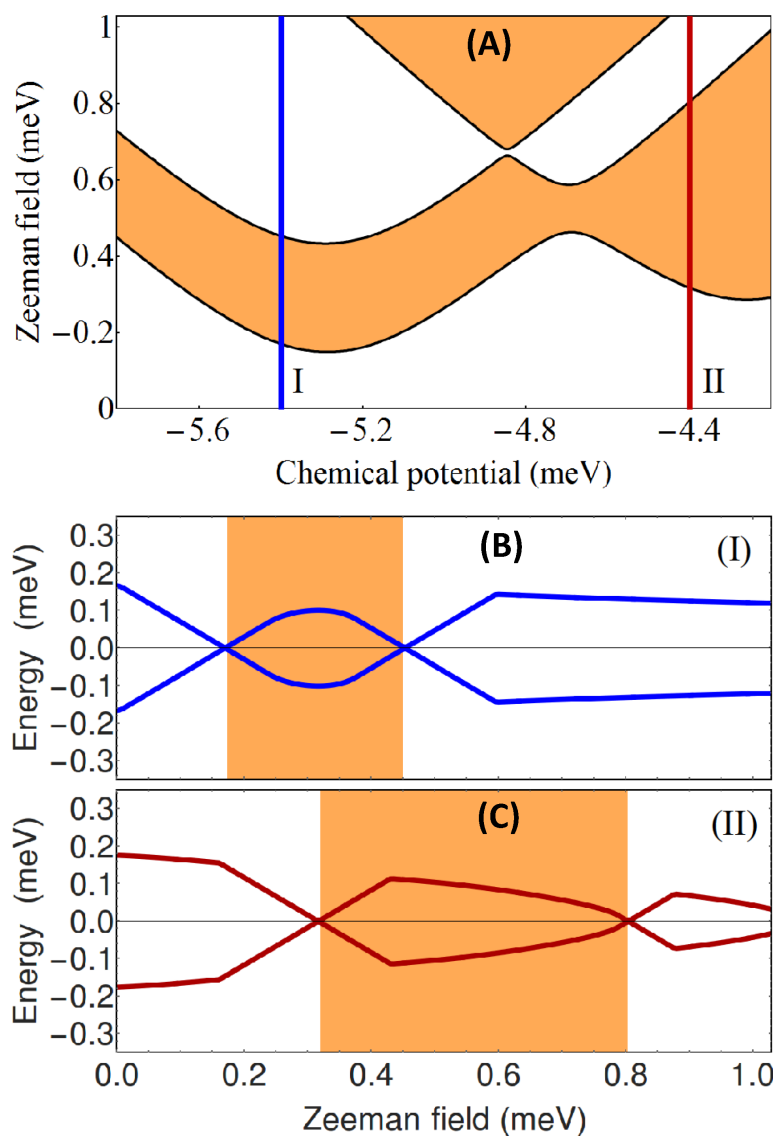

Figure 6: (A) Topological phase diagram for a triangular wire with $V_{\text {eff }}(\ell) \neq 0$ and $\phi_{1}=0, \phi_{3}=\pi / 2, \phi_{5}=-\pi / 2$. The white and orange phases are topologically trivial and nontrivial, respectively. The effective potential is the same as in Figure 2B. (B) Dependence of the minimum quasiparticle gap on the Zeeman field along the blue cut (I) in panel (A). (C) Dependence of the minimum quasiparticle gap on the Zeeman field along the dark red cut (II) in panel (A). Note the increased stability of the low-field topological phase (see for comparison Figure $2 \mathrm{~B}$ ) and the fact that the minimum critical field $\Gamma_{B}^{C} \approx 0.15 \mathrm{meV}$ is lower than the pairing potential for corner chains, $\Delta=0.3 \mathrm{meV}$. 
significant quasiparticle gap, as shown in panels (B) and (C). In addition, the lowest critical field $\Gamma_{B}^{c} \approx 0.15 \mathrm{meV}$ is about half the value of the pairing potential (i.e., $\Delta / 2$ ). This is in sharp contrast with the case of hybrid systems involving a single superconductor, or multiple superconductors having the same phase, $\phi_{\ell}=$ const., where the minimum critical field is $\Gamma_{B}^{c}=\Delta$.

A comparison between the results in Figure 2 and those in Figure 6 suggests that the superconducting phase could be used as a knob for tuning the system across a topological quantum phase transition. For example, if $\mu=-5.4 \mathrm{meV}$ and $\Gamma_{B}=0.25 \mathrm{meV}$ the system evolves as a function of the superconducting phase differences from a topologically-trivial state when $\phi_{\ell}=0$ to a topological superconductor when $\phi_{1}=0$ and $\phi_{3}=-\phi_{5}=\pi / 2$. We emphasize that the simplified tight-binding model can only provide a qualitative picture of the low-energy physics of proximitized core-shell wires. For quantitative predictions regarding the dependence of the low-energy physics on the effective bias potential $V_{\text {eff }}$ and the superconducting phases $\phi_{\ell}$ a more detailed modeling of the hybrid structure (possibly, at the microscopic level) is necessary.

To corroborate our findings regarding the effect of a phase difference, we consider the square wire corresponding to the phase diagram shown in Figure 5 coupled to four separate superconductors that induce pairing potentials characterized by $\phi_{1}=\pi / 2, \phi_{3}=-\pi / 2, \phi_{5}=\pi / 2$, and $\phi_{7}=-\pi / 2$. The corresponding phase diagram is shown in Figure 7 . The qualitative effect of having finite phase differences is the same as in the case of the triangular wire, while quantitatively it is more significant as a results of a stronger inter-chain coupling $t^{\prime}$. The topology of the phase diagram is similar to that shown in Figure 6. However, the low-field topological phase now occupies a significant region of the parameter space and the minimum critical field $\Gamma_{B}^{c}$ is practically zero. Furthermore, the topological gap is substantial, as shown in the lower panel of Figure 7, indicating a robust topological superconducting phase.

\section{Majorana modes in finite core-shell nanowires}

As a consistency check for the results discussed above, which are based on a translation-invariant model (i.e., infinite wire), and to gain further insight into the low-energy physics of the hybrid structure, we continue now with the case of wires of finite length. For concreteness, we consider a triangular wire of length $L=2.25 \mu \mathrm{m}$ in the parameter regimes corresponding to the panels labeled by "I" and "II" in Figure 3, Figure 4, and Figure 6. The dependence of the low-energy spectrum on the Zeeman field for $\mu=-5.4 \mathrm{meV}$, i.e., corresponding to the (I) panels, is shown in Figure 8. Note that when $V_{\text {eff }}=0$ and $\phi_{\ell}=0$ (top panel) the first transition is from a topologically-trivial
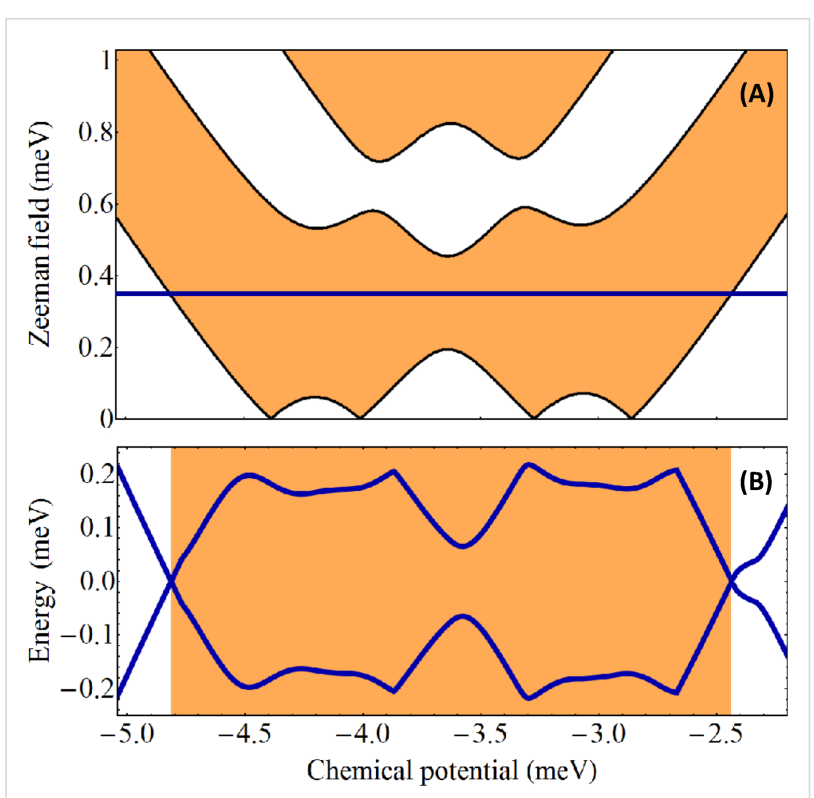

Figure 7: (A) Topological phase diagram for a square wire with $V_{\text {eff }}(\ell) \neq 0$ and $\phi_{1}=\pi / 2, \phi_{3}=-\pi / 2, \phi_{5}=\pi / 2$, and $\phi_{7}=-\pi / 2$. The white areas are topologically trivial and the orange regions are nontrivial. The values of $V_{\text {eff }}(\ell)$ and the inter-chain hopping $t^{\prime}$ are the same as in Figure 5. (B) Evolution of the minimum quasiparticle gap along the horizontal cut $\Gamma=0.35 \mathrm{meV}$ shown in the top panel. Note the significant expansion of the low-field topological phase (see for comparison Figure 5), the large topological gap, and the low values of the critical field.

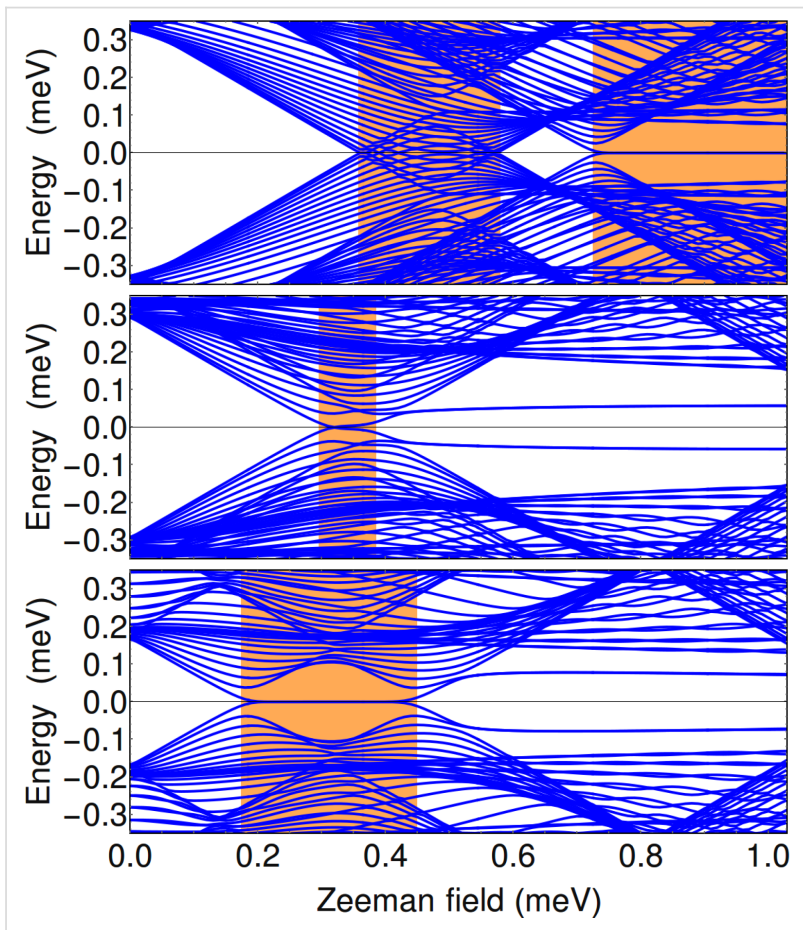

Figure 8: Dependence of the low-energy spectrum on the Zeeman field for a finite triangular wire of length $L=2.25 \mu \mathrm{m}$ and chemical potential $\mu=-5.4 \mathrm{meV}$. The parameters used in the top, middle, and bottom panels correspond to Figure $3(\mathrm{I}-\mathrm{A})$, Figure $3(\mathrm{I}-\mathrm{B})$, and Figure 6B, respectively. 
phase to a gapless superconductor, as already discussed in the context of Figure 3. The high-field topological phase $\left(\Gamma_{B}>0.7 \mathrm{meV}\right)$ is characterized by a zero-energy Majorana mode separated by a finite gap from finite energy excitations. Applying a symmetry-breaking potential $V_{\text {eff }}$ (middle panel) generates a low-field topological phase characterized by a small bulk gap and a weakly stable, energy-split Majorana mode. However, the stability of this topological phase can be significantly enhanced by creating phase differences between the parent superconductors (bottom panel). Note that in the middle and bottom panels the second trivial phase $\left(\Gamma_{B}\right.$ larger than about $0.35 \mathrm{meV}$ and $0.45 \mathrm{meV}$, respectively) is characterized by subgap states that can be viewed as pairs of overlapping, energy split Majorana bound states (at each end of the wire). This result suggests that coupling the nanowire to multiple parent superconductors and controlling their relative phases represents a powerful scheme for enhancing the robustness of the topological phase and tuning the system across a topological quantum phase transition.

The low-energy spectra for $\mu=-4.4 \mathrm{meV}$, i.e., those corresponding to the (II) panels in Figure 4 and Figure 6, are shown in Figure 9. In the top panel, note the presence of a gapless superconducting phase, which is consistent with our conclusions based on the results shown in Figure 4. Also note that the high-field topological phase $\left(\Gamma_{B}>0.85 \mathrm{meV}\right)$ supports two finite energy sub-gap modes, in addition to the zero-energy Majorana mode. Again, we can interpret these modes as pairs of overlapping Majoranas. We conclude that in this phase the hybrid system has three Majorana bound states at each end of the wire, two Majorana modes acquiring finite energy and one remaining gapless, consistent with a $\mathbb{Z}_{2}$ topological classification. Applying a symmetry-breaking potential (middle panel) enhances significantly the stability of the low-field topological phase and generates a second trivial phase $\left(\Gamma_{B}>0.9 \mathrm{meV}\right)$ that is gapped in the bulk, consistent with Figure 4. Remarkably, this trivial phase supports a pair of zero-energy Majorana modes at each end of the wire, which correspond to the mid-gap states visible in the middle panel of Figure 9. This indicates the presence of an additional "hidden" symmetry in the system, which makes it an element of the BDI symmetry class [44]. This symmetry is broken in the presence of a superconducting phase difference (bottom panel), when the sub-gap modes acquire finite energy.

\section{Symmetry and gapless superconducting phases}

The existence of the gapless superconducting phases (indicated by the star in the top panels of Figure 2 and Figure 3) is a consequence of the threefold rotation symmetry of the triangular wire with $V_{\text {eff }}(\ell)=0$ and identical superconductors.

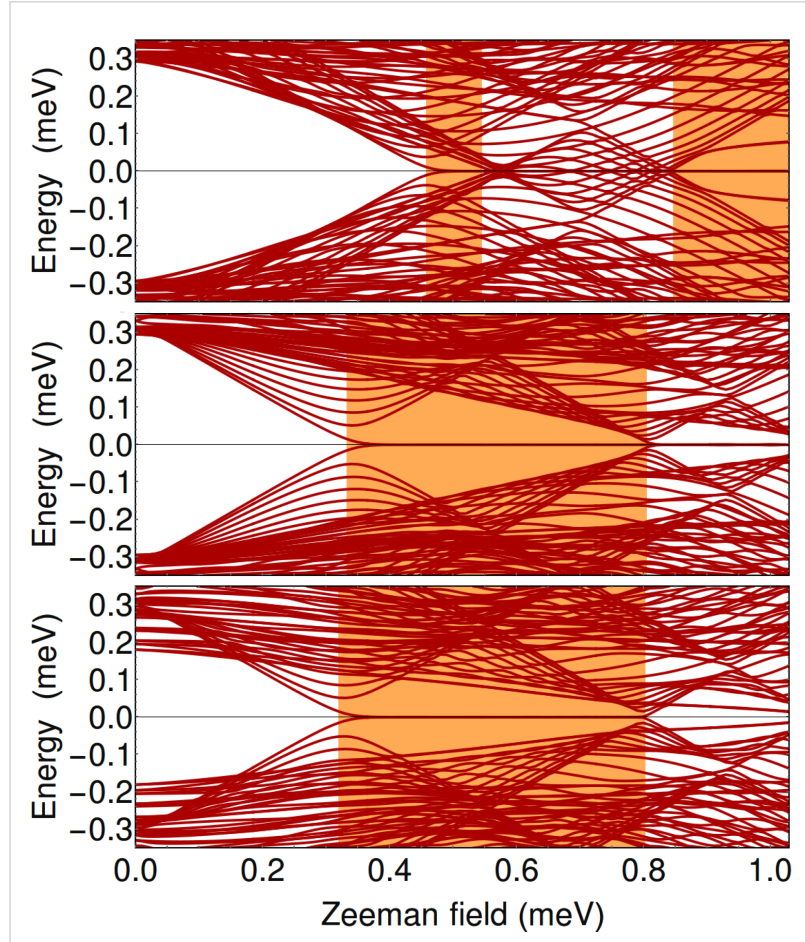

Figure 9: Dependence of the low-energy spectrum on the Zeeman field for a finite triangular wire of length $L=2.25 \mu \mathrm{m}$ and chemical potential $\mu=-4.4 \mathrm{meV}$. The parameters used in the top, middle, and bottom panels correspond to Figure 4(II-A), Figure 4(II-B), and Figure $6 \mathrm{C}$, respectively.

Breaking this symmetry automatically opens a (bulk) gap in the spectrum. To illustrate this property we consider the system of finite length $L=2.25 \mathrm{~nm}$, with the other parameters corresponding to Figure $2 \mathrm{~A}$, with chemical potential $\mu=-5.4 \mathrm{meV}$ (i.e., the blue vertical line there), and $V_{\text {eff }}(\ell)=0$, and we focus on the gapless phase $0.36<\Gamma_{B}<0.58 \mathrm{meV}$. The low-energy spectrum is shown in Figure 10A, which is in fact a zoom into the top panel of Figure 8. We consider now a small symmetrybreaking potential, with the same proportions as in Figure 2B, Figure 3(I-B), and middle panel of Figure 8, but now ten times weaker, i.e., $V_{\text {eff }}=V_{0}(2,0.5,-1,-1,-1,0.5)$ with $V_{0}=33.3 \mu \mathrm{eV}$. The potential opens a bulk gap that hosts a midgap Majorana mode, as shown in Figure 10B. To emphasize that the opening of a bulk gap is the result of breaking the threefold rotation symmetry, we also consider a system with vanishing effective potential, $V_{\text {eff }}(\ell)=0$, in which we break the symmetry by coupling the wire to parent superconductors having different bulk gaps, so that the proximity-induced pairing potentials for the edges are $\Delta_{1}=0.375 \mathrm{meV}$, $\Delta_{3}=0.300 \mathrm{meV}$, and $\Delta_{5}=0.300 \mathrm{meV}$. Here we do not consider any relative phase between the superconductors. Again, a small bulk gap opens in the (bulk) spectrum and a (nearly-zero) Majorana mode emerges as a mid-gap state, as can be seen Figure 10C. 


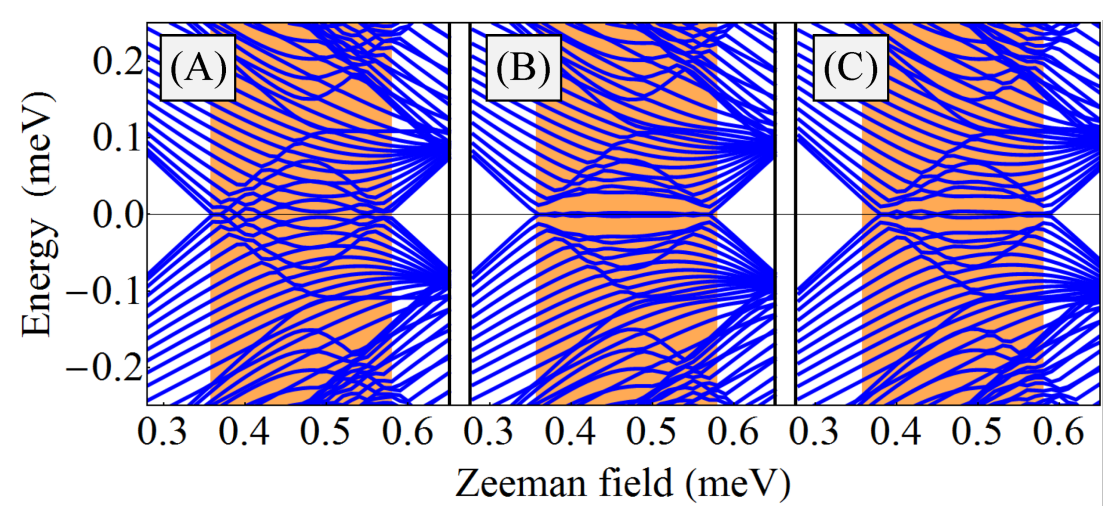

Figure 10: Low-energy spectra as a function of the Zeeman field for a finite triangular wire of length $L=2.25 \mu \mathrm{m}$ and chemical potential $\mu=-5.4 \mathrm{meV}$. (A) Gapless superconducting phase in a system with threefold rotation symmetry, like in Figure 2A and Figure 3(I-A). (B) Applying a symmetry-breaking $V_{\text {eff, }}$ ten times waker than in Figure $2 B$, a small bulk gap develops, like in Figure 3(I-B), that hosts a mid-gap Majorana mode. (C) Symmetry broken by coupling the wire to different superconductors inducing edge pairing potentials $\Delta_{1}=0.375 \mathrm{meV}, \Delta_{3}=0.3 \mathrm{meV}$, and $\Delta_{5}=0.3$ $\mathrm{meV}$. The filled (orange) region $0.36<\Gamma_{B}<0.58 \mathrm{meV}$ represents the topological superconducting phase (of an infinite wire) in the presence of an infinitesimally-small symmetry-breaking perturbation.

Another important general property of the Majorana modes illustrated in Figure 10, panels (B) and (C), is the presence of energy splitting oscillations $[25,45]$. In general, the energy splitting is caused by a finite overlap of the Majorana modes localized at the opposite ends of the wire. The amplitude of the oscillations depends on the Majorana localization length $\xi$ [25], which increases as the topological gap decreases, diverging in the gapless limit. This behavior is illustrated in Figure 11. The top panel represents the lowest-energy state corresponding to a gapless system with threefold rotation symmetry (i.e., $V_{\text {eff }}=0$ ), which could be seen as a linear combination of Majorana modes with an infinite characteristic lenghscale, $\xi \rightarrow \infty$. Introducing a symmetry-breaking perturbation $\left(V_{\text {eff }} \neq 0\right)$ opens a (bulk) topological gap that increases with increasing the effective potential. In addition, in a finite system a midgap state emerges, consisting of two (partially) overlapping Majorana modes localized at the opposite ends of the wire. As clearly shown in Figure 11, the characteristic length scale $\xi$ of the Majorana modes decreases as the amplitude $V_{0}$ of the symmetry-breaking potential increases (i.e., as the topological gap increases).

We note that, from the perspective of quantum computation, the zero-energy Majorana modes have to be i) well separated spatially (to minimize the overlap and, consequently, the energy splitting $\delta E$ ) and ii) well separated in energy from all other lowenergy states (by a certain minimum quasiparticle gap $\Delta E$ ). The first condition ensures that the Majorana modes have nonAbelian properties, while the second guarantees that the parity of the low-energy Majorana sub-space is fixed (the presence of other low-energy states would allow excitations from the Majorana sub-space, which would change its parity and destroy any quantum information stored in the Majorana system). If these conditions are satisfied, the Majorana modes span a nearly-zero

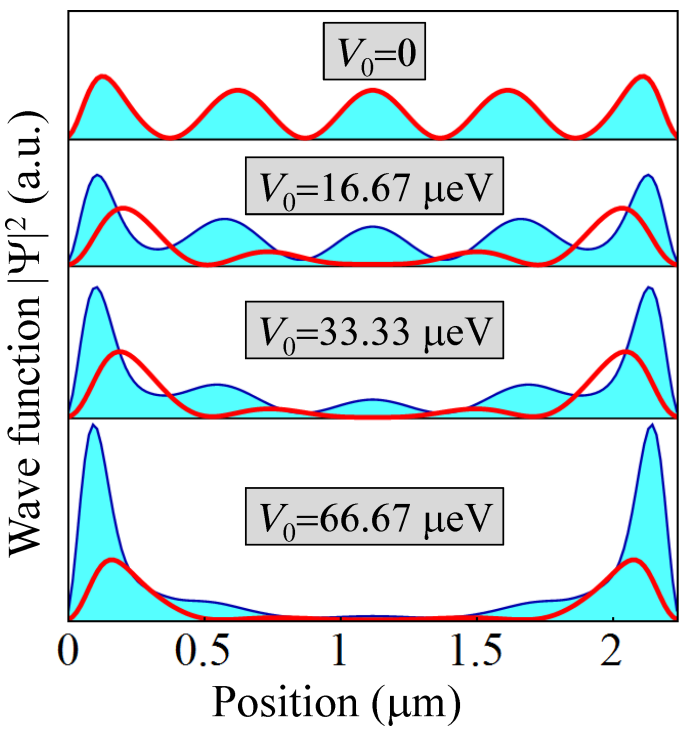

Figure 11: Position dependence of the lowest energy wave function corresponding to a finite triangular wire of length $L=2.25 \mu \mathrm{m}$, chemical potential $\mu=-5.4 \mathrm{meV}$, Zeeman field $\Gamma_{B}=0.45 \mathrm{meV}$, and symmetry-breaking effective potential with amplitude $V_{0}$ (see Figure 10B). The thick (red) line represents the probability distribution $\left|\Psi_{1}(x)\right|^{2}$ along the edge $\ell=1$, while the filled (blue) line represents the probability distribution along the edges $\ell=3,5$. With increasing the amplitude of the symmetry-breaking potential the (bulk) topological gap increases, which leads to the reduction of the characteristic length $\xi$ of the Majorana modes localized at the opposite ends of the wire.

energy subspace that can be used for storing and processing quantum information. The characteristic timescale $\tau$ for quantum operations has to satisfy the condition $\delta E \ll \hbar / \tau \ll \Delta E$. Of course, the impossibility of satisfying this condition is manifest in regimes characterized by small topological gaps, as $\delta E$ and $\Delta E$ become comparable in the gapless superconductor limit. 


\section{Effects of disorder}

Another element that can compromise the topological protection of the Majorana subspace is the presence of disorder. Generically, disorder induces low-energy sub-gap states, thus reducing $\Delta E[46-50]$. The effect of potential disorder on a topological phase realized in a triangular wire is illustrated in Figure 12. Panel (A) shows the position dependence (along the wire) of a typical disorder potential $V_{\text {dis }}(x)$ considered in the calculation. Next, we calculate the low-energy spectrum in the presence of a disorder potential with a fixed profile but a varying amplitude $V_{\max }$ (see Figure 12B). As the disorder strength increases, several low-energy states converge toward zero-energy, so that the quasiparticle gap $\Delta E$ practically collapses when the amplitude of the effective disorder potential is larger than $V_{\text {max }} \approx 1 \mathrm{meV}$. To demonstrate that this is not an accidental property of a specific disorder realization, we also calculated the spectrum averaged over multiple disorder realizations (see Figure 12C). The qualitative features discussed above are manifestly present. We note that "critical" disorder strength
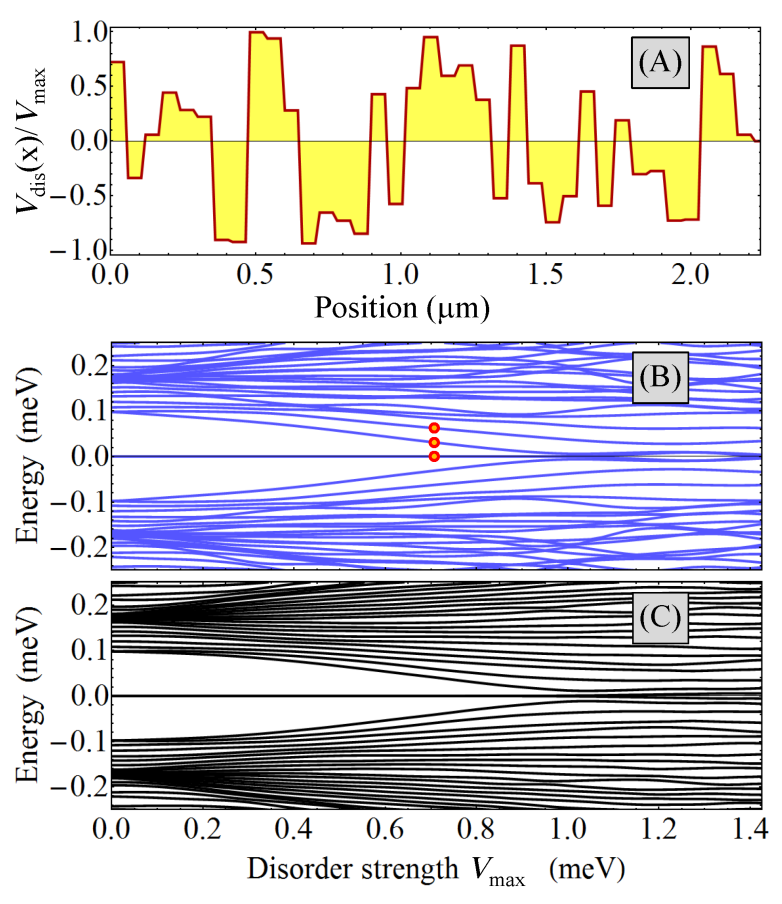

Figure 12: (A) Position dependence of the normalized disorder potential along the edge $\ell=3$ of a triangular wire for a specific disorder realization. The disorder profiles along the edges $\ell=1,5$ (not shown) are different, but characterized by similar qualitative features. In particular, the characteristic length scale for the potential variations is $\delta_{d}=60 \mathrm{~nm}$. (B) Dependence of the low-energy spectrum on the amplitude $V_{\max }$ of the disorder potential for the disorder realization shown in panel (A).

(C) Low-energy spectrum averaged over 50 different disorder realizations as a function of $V_{\max }$. The parameters of the system are: wire length $L=2.25 \mu \mathrm{m}$, chemical potential $\mu=-5.4 \mathrm{meV}$, effective potential $V_{\text {eff }}=(0.67,0.17,-0.33,-0.33,-0.33,0.17) \mathrm{meV}$, superconducting phases $\phi_{1}=0, \phi_{3}=\pi / 2, \phi_{5}=-\pi / 2$ and Zeeman field $\Gamma_{B}=0.35 \mathrm{meV}$ associated with the collapse of the quasiparticle gap depends on the characteristic length scale of the disorder potential, as well as the topological gap of the clean system, larger gaps implying an increased robustness against disorder.

The final point that we want to address concerns the structure of the disorder-induced low-energy states. Specifically, we calculate the spatial profiles of the three lowest-energy states marked by red dots in Figure 12B. The results are shown in Figure 13. We note that the Majorana modes $(n=1)$ are well localized near the opposite ends of the wire and have most of the spectral weight on the edges $\ell=3,5$ as a result of applying a bias potential $V_{\text {eff }}(\ell)$. The disorder-induced states $(n=2,3)$ are localized inside the wire and have most of their spectral weight on the same edges, $\ell=3,5$. We conclude that the presence of disorder induces low-energy localized states than can destroy the topological protection of the Majorana subspace. We note that within a topological quantum computation scheme based on qubits characterized by a finite charging energy [51,52], interaction-mediated transitions between the Majorana modes and disorder-induced localized states are possible even when the spatial overlap of the two types of states is exponentially small. Such transitions, which create low-energy quasiparticles, could completely compromise the topological protection of the quantum computation scheme.

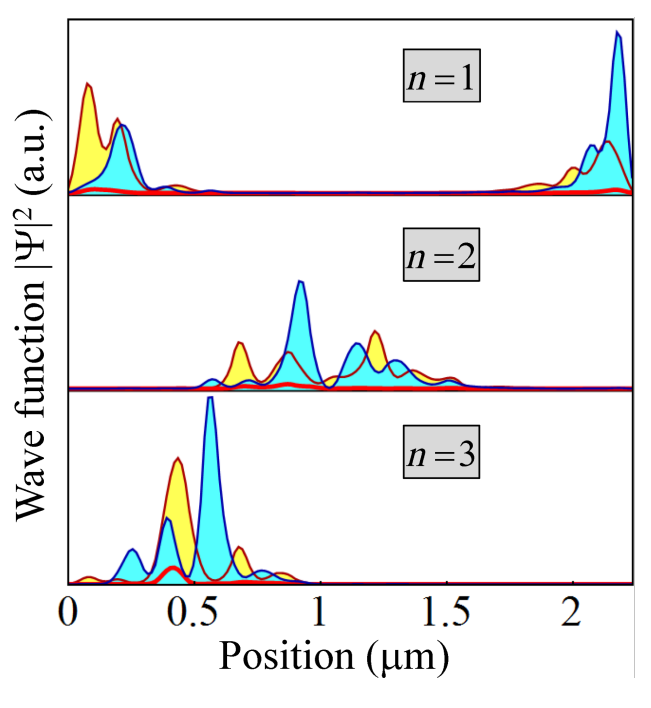

Figure 13: Spatial profiles of the three lowest energy states corresponding to the red dots in Figure 12B. The thick (red) line represents the profile along the edge $\ell=1$, while the filled lines represent the profiles along the edge $\ell=3$ (blue/light blue filling) and $\ell=5$ (dark red/yellow filling).

\section{Geometrical model of a prismatic shell}

In this section we analyze the results of a finer-grained model of triangular and square prismatic shells, based on a geometrical 
description [29]. First the two-dimensional Hamiltonian of a single electron confined on the polygonal cross section is discretized on a grid defined in polar coordinates and diagonalized numerically $[37,53]$. The resulting low-energy eigenstates, corresponding to corner localization, are further used as a basis to find the eigenstates of the BdG Hamiltonian, assuming plane waves in direction longitudinal to the prism. The basis includes the spin and the isospin. The variable Zeeman energy is generated by a uniform magnetic field $B$ longitudinal to the wire. In addition we consider a relatively weak electric field $E$ transverse to the wire as a technical tool to break the symmetry of the polygon, indicated by the red arrows in Figure 14. This field is equivalent with the chain dependent potential $V_{\text {eff }}(\ell)$ introduced before. First, a perfectly symmetric shell is experimentally unrealistic from fabrication. Second, as already mentioned, in a regular experimental setup external gates and other contacts may affect the wire symmetries. Third, a generic electric field can be seen as a tunable parameter that can change the topological phase diagram.
(A)

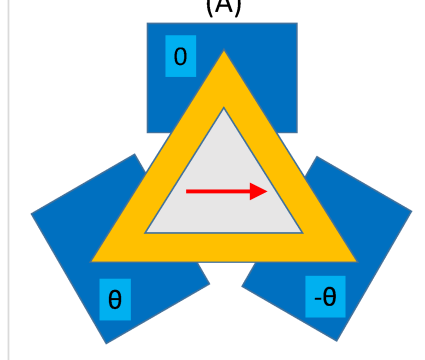

(B)

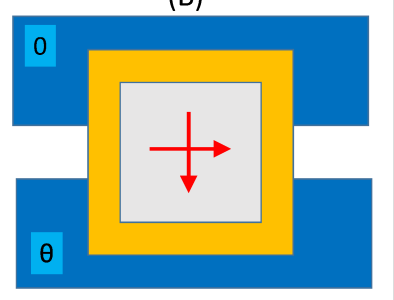

Figure 14: A schematic cross section of the hybrid semiconductorsuperconductor experimental device incorporating a core-shell wire. The core is shown in grey and the shell in yellow. The blue blocks represent the superconductor metals attached to the wire. The lower superconductors can have phases $\pm \theta$ relatively to the upper one considered with zero phase. The red arrows indicate the electric field included in our geometrical model. (A) In the triangular case it is parallel to one side of the triangle. (B) In the square case it can be either perpendicular or parallel to the superconductors.

We characterize the lateral size of the wire with the radius $R$ of a circle surrounding the shell, and with the shell thickness $d$. In the present calculations we use $R=50 \mathrm{~nm}$ for both geometries, but $d=12.5 \mathrm{~nm}$ for the triangular shell and $d=8 \mathrm{~nm}$ for the square shell. These values are comparable to the dimensions of the realistic core-shell nanowires mentioned in the experimental papers [32-36]. The material parameters of the shell are chosen as for InSb. For these geometric parameters and with $m_{\text {eff }}=0.014$ the energy separation between the corner and side states is about 41 and $38 \mathrm{meV}$ for the triangular and square case, respectively, meaning that for these parameters the low energy physics can be very well described by the corner states. Therefore we can use a Rashba SOC model similar to that of the planar electron gas, but on a cylindrical surface of radius $R$, i.e., transformed from Cartesian to polar coordinates [54]. Since the sides of the triangular shell are unpopulated this model is qualitatively reasonable, and can lead to Majorana states. As mentioned before a more elaborated microscopic description of the SOC is beyond the scope of the present paper, and here we simply adopt in the numerical calculations the coupling constant of bulk InSb, of $50 \mathrm{meV} / \mathrm{nm}$.

For a symmetric triangle the corner states have equal probability distribution at each corner [37], whereas in the presence of a weak electric field $E$, here corresponding to $0.22 \mathrm{mV}$ across the radius $R$, they separate. The wave functions still have some exponential tails along the sides of the polygon, which are equivalent to the inter-chain hopping introduced earlier. The phase diagram shown in Figure 15A is obtained with a real valued superconductor gap $\Delta=0.5 \mathrm{meV}$, and can be compared with Figure 2B (where all $\phi_{\ell}=0$ ). The fragmentation of the phase boundaries in three dark lines reflects the presence of the three corners (edges) of the prismatic wire. The boundaries approach each other when the aspect ratio of the triangle $(d / R)$ decreases, which results in reduced overlap of the wave functions of the corner states [29].

The colors used indicate the minimum gap of the BdG spectrum at any wave vector $k$, on a logarithmic scale, so the representation is complementary to the two-color scheme of Figure 2B (or A). Here the topological phases can be identified by the number of crossings of the dark lines. Along these lines the gap closes at $k=0$. Starting from any point outside the boundaries one enters into a topological Majorana phase after the first intercept of a dark line, then into the trivial phase after the second intercept, and again into the topological phase after the third intercept.

Next, in Figure 15B, we show the phase diagram obtained with a complex valued superconductor gap, of constant modulus and variable phases, which are zero at one corner and $\pm \pi / 6$ at the other corners (i.e., $\theta=\pi / 6$ in Figure 14A). We obtain a splitting (or anticrossing) of the phase boundaries at the former crossing point, similar to that shown in Figure 6A, although now more pronounced than in the chain model.

By further increasing the relative (angular) phase $\theta$ to $\pm \pi / 2$ the boundaries of the quantum phase transitions become nearly parallel, Figure 15C. This result can be interpreted as an increased interaction between the corner states in the presence of the phase shift $\theta$ of the superconductors. Another consequence of this phase shift is that the absolute gap of the BdG spectrum decreases in some topological regions, as indicated by the diffuse reddish regions, suggesting that some topological states 


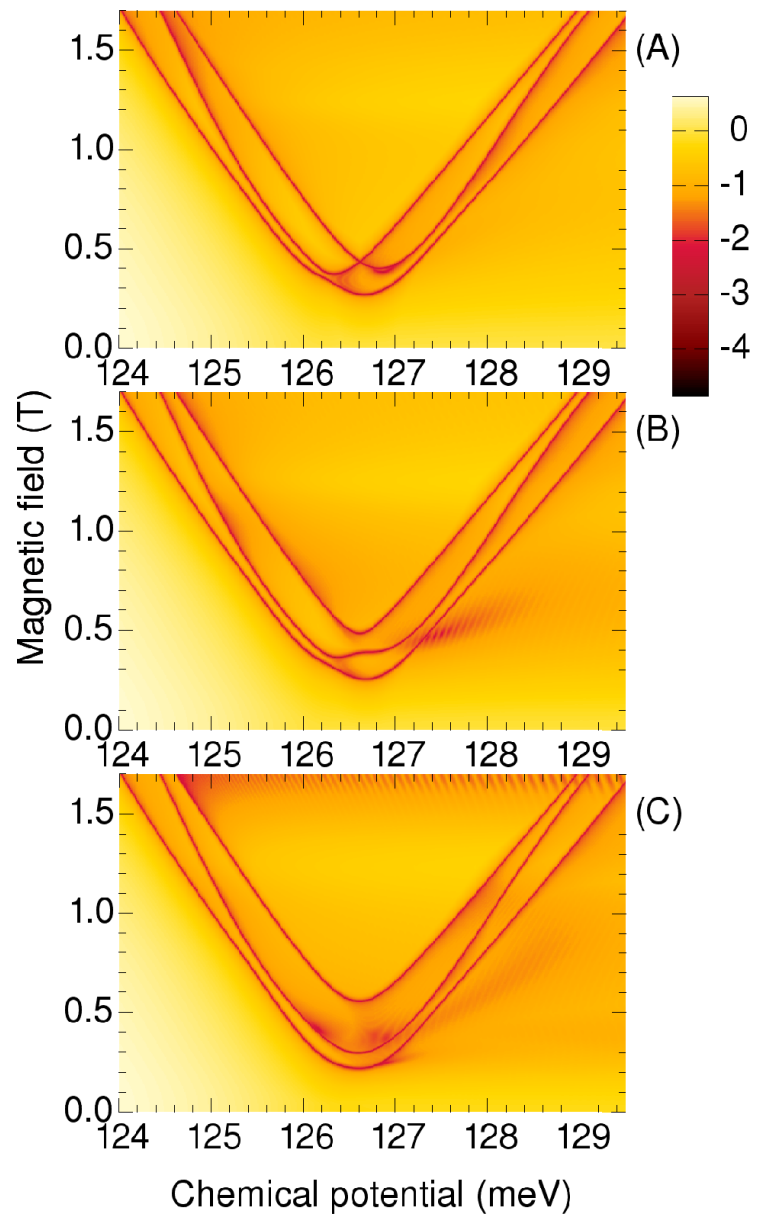

Figure 15: Phase boundaries for the triangular wire in the corner-state domain. The color code describes the minimum gap of the BdG spectra for all wave vectors. The character of each phase can be identified by counting the boundary crossings along a vertical line, starting at zero magnetic field, i.e., topological or trivial for an odd or an even number of crossings, respectively. Along these boundaries the gap closes at $k=0$. Starting from any point outside the (A) All superconductor phases are equal to zero. (B) Phases are: 0 at one corner and $\pm \pi / 6$ at the other corners, i.e., $\theta=\pi / 6$ in Figure 14A. (C) The same phase distribution, with $\theta=\pi / 2$.

may become gapless. This tendency is consistent with the results of the multiple chain model, compare Figure 4B with Figure 6C.

As with the coupled-chains model, we also tested the effect of using two superconductors with different gaps, for example by reducing the gap parameter $\Delta$ of one or two superconductors by one half, and using no relative phase, $\theta=0$. The resulting phase diagrams were qualitatively like those shown in Figure 15B,C, although with lower energy gaps in the topological phases. This indicates no particular gain by creating an asymmetry in this way, compared to using the superconductors with the large gap and creating the asymmetry via the relative phase $\theta$.
Finally, in Figure 16 we show the phase diagrams obtained with the geometric model for the square shell profile. Here, in the geometrical model, we use a particular setup for the square geometry, with only two superconductors. Unlike in the coupledchains model, in this case the superconductors are also connected to the states localized on the sides of the polygon, if those states would be populated, but this is not the case for the chemical potentials used for Figure 16. First we note that we obtain four phase boundaries, according to the presence of four corner states. As for the triangular geometry the trivial or topological character of the phases is associated with odd or even number of boundary crossings, respectively, when starting from the outer regions. Therefore the central zone of the phase diagrams is now topologically trivial. In Figure 16A we show

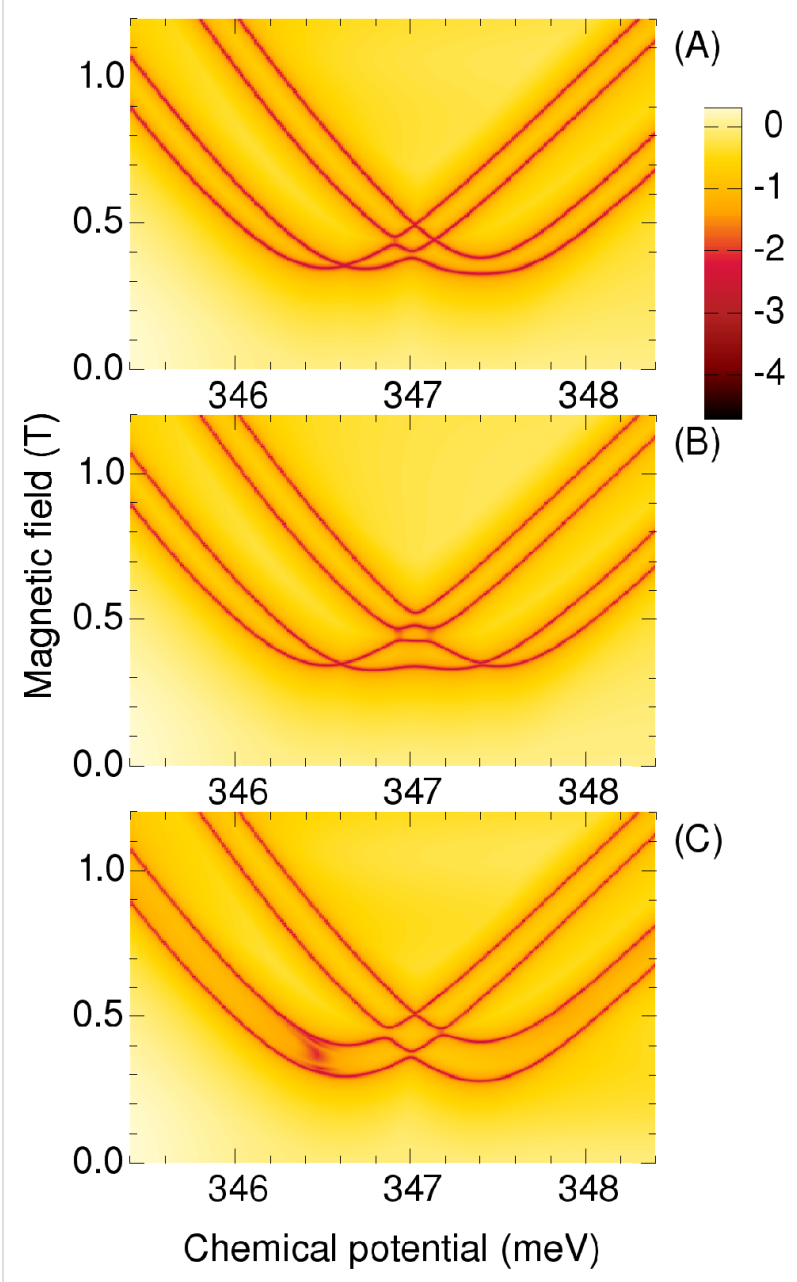

Figure 16: Phase boundaries for the square wire in the corner-state domain. The color code describes the minimum gap of the BdG spectra for all wave vectors. The topological or trivial character of the phases can be identified by the number of boundary crossings, as described in the caption of Figure 15. (A) The superconductor phases equal to zero. (B) The superconductor phases are zero and $\theta=\pi / 2$, and the electric field perpendicular to the superconductors, see Figure 14B. (C) Again $\theta=\pi / 2$, but with the electric field parallel to the superconductors. 
the results with $\theta=0$, i.e., no phase shift between the superconductors (Figure 14B). The electric field corresponds now to $60 \mathrm{mV}$ per radius, and obviously the results do not depend on the two orientation considered here if $\theta=0$.

Remarkably, with a finite phase shift, here $\theta=\pi / 2$, the phase diagrams are different when the electric field is perpendicular, Figure 16B, or parallel to the superconductors, Figure 16C, respectively. In the perpendicular case the phase frontiers are mostly changed in the central region, whereas in the parallel case they are more affected in the low field part. In the first case the corner states with phase $\theta$ are separated energetically from those with zero phase, but they still interact when they are all grouped within or close to the superconductor gap. In the second case the states with the same superconductivity phase are separated, and the frontiers tend to become parallel.

\section{Conclusion}

In this work we have studied the phase diagram of core-shell nanowires coupled with multiple parent superconductors using a simplified tight-binding parallel-chain model. We found that applying a potential that breaks the (intrinsic) rotation symmetry of the wire does not modify the topology of the phase diagram, but removes the gapless superconducting phases that populate certain regions of the phase diagram and partially stabilizes the topological superconducting phase. Remarkably, finite phase differences between the parent superconductors have dramatic effects. First, the topology of the phase diagram is modified. In particular the "crossing points" that characterize the phase diagram in the presence of a uniform superconducting phase disappear and, upon increasing the Zeeman field, we have an alternance of trivial and nontrivial phases for all values of the chemical potential. More importantly, the lowfield topological phase becomes stable for a wide range of chemical potentials and the minimum critical field $\Gamma_{B}^{c}$ can have arbitrarily low values. We conclude that by controlling the relative phases of the parent superconductors coupled to the wire one can stabilize the topological superconducting phase that hosts the zero-energy Majorana modes and one can obtain a powerful additional experimental knob for exploring a rich phase diagram and observing potentially interesting low-energy physics. Given the potential experimental significance of these conclusions, we believe that a more detailed and systematic investigation of these effects, which is beyond the goal of the present work, would be warranted.

In particular, the effect of electrostatic interactions on the properties of the normal electronic states in core-shell nanowires can be important. The effect of interactions should be calculated using a Schrödinger-Poisson scheme, e.g., like in [55], to take into account both the interface potential between the core and the shell, and the presence of the carrier density in the shell. In addition, for Majorana devices, one should incorporate the effects due to the presence of a parent superconductor, including the work function difference between the superconductor and the semiconductor, as well as the effects generated by gateinduced electric fields. An efficient method for implementing the Schrödinger-Poisson scheme in calculations using realistic three-dimensional models of hybrid devices has been recently proposed in [56]. We emphasize that, due to the corner and side localization, the electron-electron interactions have nontrivial effects [57], which can modify the proximity-induced superconductor gap and the phase diagram of the Majorana states [5865]. The calculation of the effective potential profile is also essential for estimating the SOC in the nanowire. Therefore, accounting for the electrostatic effects represents a key step toward a quantitative theory of Majorana physics in core-shell nanowires.

\section{Acknowledgements}

This work was partially financed by the research funds of Reykjavik University and by the Icelandic Research Fund. TDS was supported in part by NSF DMR-1414683.

\section{ORCID ${ }^{\circledR}$ iDs}

Andrei Manolescu - https://orcid.org/0000-0002-0713-4664

\section{References}

1. Kitaev, A. Yu. Phys.-Usp. 2001, 44, 131. doi:10.1070/1063-7869/44/10S/S29

2. Kitaev, A. Yu. Ann. Phys. 2003, 303, 2. doi:10.1016/S0003-4916(02)00018-0

3. Wilczek, F. Nat. Phys. 2009, 5, 614. doi:10.1038/nphys 1380

4. Stanescu, T. D. Introduction to topological quantum matter and quantum computation; CRC Press: Boca Raton, FL, U.S.A., 2017.

5. Sau, J. D.; Lutchyn, R. M.; Tewari, S.; Das Sarma, S. Phys. Rev. Lett. 2010, 104, 040502. doi:10.1103/PhysRevLett.104.040502

6. Alicea, J. Phys. Rev. B 2010, 81, 125318. doi:10.1103/physrevb.81.125318

7. Oreg, Y.; Refael, G.; von Oppen, F. Phys. Rev. Lett. 2010, 105, 177002. doi:10.1103/PhysRevLett.105.177002

8. Lutchyn, R. M.; Sau, J. D.; Das Sarma, S. Phys. Rev. Lett. 2010, 105, 077001. doi:10.1103/PhysRevLett.105.077001

9. Sau, J. D.; Tewari, S.; Lutchyn, R. M.; Stanescu, T. D.; Das Sarma, S. Phys. Rev. B 2010, 82, 214509. doi:10.1103/PhysRevB.82.214509

10. Alicea, J. Rep. Prog. Phys. 2012, 75, 076501. doi:10.1088/0034-4885/75/7/076501

11. Stanescu, T. D.; Tewari, S. J. Phys.: Condens. Matter 2013, 25 , 233201. doi:10.1088/0953-8984/25/23/233201

12. Beenakker, C. W. J. Annu. Rev. Condens. Matter Phys. 2013, 4, 113. doi:10.1146/annurev-conmatphys-030212-184337

13. Franz, M. Nat. Nanotechnol. 2013, 8, 149. doi:10.1038/nnano.2013.33

14. Mourik, V.; Zuo, K.; Frolov, S. M.; Plissard, S. R.; Bakkers, E. P. A. M.; Kouwenhoven, L. P. Science 2012, 336, 1003.

doi:10.1126/science. 1222360 
15. Deng, M. T.; Yu, C. L.; Huang, G. Y.; Larsson, M.; Caroff, P.; Xu, H. Q. Nano Lett. 2012, 12, 6414. doi:10.1021/nl303758w

16. Das, A.; Ronen, Y.; Most, Y.; Oreg, Y.; Heiblum, M.; Shtrikman, H. Nat. Phys. 2012, 8, 887. doi:10.1038/nphys2479

17. Finck, A. D. K.; Van Harlingen, D. J.; Mohseni, P. K.; Jung, K.; Li, X. Phys. Rev. Lett. 2013, 110, 126406. doi:10.1103/PhysRevLett.110.126406

18. Churchill, H. O. H.; Fatemi, V.; Grove-Rasmussen, K.; Deng, M. T.; Caroff, P.; Xu, H. Q.; Marcus, C. M. Phys. Rev. B 2013, 87, 241401. doi:10.1103/PhysRevB.87.241401

19. Albrecht, S. M.; Higginbotham, A. P.; Madsen, M.; Kuemmeth, F.; Jespersen, T. S.; Nygård, J.; Krogstrup, P.; Marcus, C. M. Nature 2016, 531, 206. doi:10.1038/nature17162

20. Deng, M. T.; Vaitiekènas, S.; Hansen, E. B.; Danon, J.; Leijnse, M.; Flensberg, K.; Nygård, J.; Krogstrup, P.; Marcus, C. M. Science 2016, 354, 1557. doi:10.1126/science.aaf3961

21. Chen, J.; Yu, P.; Stenger, J.; Hocevar, M.; Car, D.; Plissard, S. R.; Bakkers, E. P. A. M.; Stanescu, T. D.; Frolov, S. M. Sci. Adv. 2017, 3, e1701476. doi:10.1126/sciadv.1701476

22. Zhang, H.; Gül, O.; Conesa-Boj, S.; Nowak, M. P.; Wimmer, M.; Zuo, K.; Mourik, V.; de Vries, F. K.; van Veen, J.; de Moor, M. W.; Bommer, J. D.; van Woerkom, D. J.; Car, D.; Plissard, S. R.; Bakkers, E. P.; Quintero-Perez, M.; Cassidy, M. C.; Koelling, S.; Goswami, S.; Watanabe, K.; Taniguchi, T.; Kouwenhoven, L. P. Nat. Commun. 2017, 8, 16025. doi:10.1038/ncomms16025

23. Nichele, F.; Drachmann, A. C. C.; Whiticar, A. M.; O'Farrell, E. C. T.; Suominen, H. J.; Fornieri, A.; Wang, T.; Gardner, G. C.; Thomas, C.; Hatke, A. T.; Krogstrup, P.; Manfra, M. J.; Flensberg, K.; Marcus, C. M. Phys. Rev. Lett. 2017, 119, 136803. doi:10.1103/PhysRevLett.119.136803

24. Zhang, H.; Liu, C.-X.; Gazibegovic, S.; Xu, D.; Logan, J. A.; Wang, G.; van Loo, N.; Bommer, J. D.; de Moor, M. W.; Car, D.; het Veld, R. L. M. O.; van Veldhoven, P. J.; Koelling, S.; Verheijen, M. A.; Pendharkar, M.; Pennachio, D. J.; Shojaei, B.; Lee, J. S.; Palmstrom, C. J.; Bakkers, E. P.; Sarma, S. D.; Kouwenhoven, L. P. arXiv 2017, No. 1712.08459.

25. Das Sarma, S.; Sau, J. D.; Stanescu, T. D. Phys. Rev. B 2012, 86, 220506. doi:10.1103/PhysRevB.86.220506

26. Lutchyn, R. M.; Bakkers, E. P. A. M.; Kouwenhoven, L. P.; Krogstrup, P.; Marcus, C. M.; Oreg, Y. arXiv 2017, No. 1707.04899. And references therein.

27. Ihn, T. Semiconductor Nanostructures: Quantum states and electronic transport; Oxford Scholarship Online: Oxford, United Kingdom, 2010.

28. Nijholt, B.; Akhmerov, A. R. Phys. Rev. B 2016, 93, 235434. doi:10.1103/PhysRevB.93.235434

29. Manolescu, A.; Sitek, A.; Osca, J.; Serra, L.; Gudmundsson, V.; Stanescu, T. D. Phys. Rev. B 2017, 96, 125435. doi:10.1103/PhysRevB.96.125435

30. Bertoni, A.; Royo, M.; Mahawish, F.; Goldoni, G. Phys. Rev. B 2011, 84, 205323. doi:10.1103/PhysRevB.84.205323

31. Hu, M.; Zhang, X.; Giapis, K. P.; Poulikakos, D. Phys. Rev. B 2011, 84, 085442. doi:10.1103/PhysRevB.84.085442

32. Wong, B. M.; Léonard, F.; Li, Q.; Wang, G. T. Nano Lett. 2011, 11, 3074. doi:10.1021/nl200981x

33. Blömers, C.; Rieger, T.; Zellekens, P.; Haas, F.; Lepsa, M. I.; Hardtdegen, H.; Gül, Ö.; Demarina, N.; Grützmacher, D.; Lüth, H.; Schäpers, T. Nanotechnology 2013, 24, 035203. doi:10.1088/0957-4484/24/3/035203
34. Qian, F.; Brewster, M.; Lim, S. K.; Ling, Y.; Greene, C.; Laboutin, O.; Johnson, J. W.; Gradečak, S.; Cao, Y.; Li, Y. Nano Lett. 2012, 12, 3344. doi:10.1021/nl301690e

35. Heurlin, M.; Stankevič, T.; Mickevičius, S.; Yngman, S.; Lindgren, D.; Mikkelsen, A.; Feidenhans, R.; Borgstöm, M. T.; Samuelson, L. Nano Lett. 2015, 15, 2462. doi:10.1021/nl5049127

36. Yuan, X.; Caroff, P.; Wang, F.; Guo, Y.; Wang, Y.; Jackson, H. E.; Smith, L. M.; Tan, H. H.; Jagadish, C. Adv. Funct. Mater. 2015, 25, 5300. doi:10.1002/adfm.201501467

37. Sitek, A.; Serra, L.; Gudmundsson, V.; Manolescu, A. Phys. Rev. B 2015, 91, 235429. doi:10.1103/PhysRevB.91.235429

38. Pöyhönen, K.; Westström, A.; Röntynen, J.; Ojanen, T. Phys. Rev. B 2014, 89, 115109. doi:10.1103/PhysRevB.89.115109

39. Wakatsuki, R.; Ezawa, M.; Nagaosa, N. Phys. Rev. B 2014, 89, 174514. doi:10.1103/PhysRevB.89.174514

40. Wimmer, M. ACM Trans. Math. Softw. 2012, 38, 30. doi:10.1145/2331130.2331138

41. Ghosh, P.; Sau, J. D.; Tewari, S.; Das Sarma, S. Phys. Rev. B 2010, 82, 184525. doi:10.1103/PhysRevB.82.184525

42. Pientka, F.; Keselman, A.; Berg, E.; Yacoby, A.; Stern, A.; Halperin, B. I. Phys. Rev. X 2017, 7, 021032. doi:10.1103/PhysRevX.7.021032

43. Sitthison, P.; Stanescu, T. D. Phys. Rev. B 2014, 90, 035313. doi:10.1103/PhysRevB.90.035313

44. Dumitrescu, E.; Stanescu, T. D.; Tewari, S. Phys. Rev. B 2015, 91, 121413. doi:10.1103/PhysRevB.91.121413

45. Cheng, M.; Lutchyn, R. M.; Galitski, V.; Das Sarma, S. Phys. Rev. Lett. 2009, 103, 107001. doi:10.1103/PhysRevLett.103.107001

46. Bagrets, D.; Altland, A. Phys. Rev. Lett. 2012, 109, 227005. doi:10.1103/PhysRevLett.109.227005

47. Liu, J.; Potter, A. C.; Law, K. T.; Lee, P. A. Phys. Rev. Lett. 2012, 109, 267002. doi:10.1103/PhysRevLett.109.267002

48. DeGottardi, W.; Sen, D.; Vishveshwara, S. Phys. Rev. Lett. 2013, 110, 146404. doi:10.1103/PhysRevLett.110.146404

49. Rainis, D.; Trifunovic, L.; Klinovaja, J.; Loss, D. Phys. Rev. B 2013, 87, 024515. doi:10.1103/PhysRevB.87.024515

50. Adagideli, I.; Wimmer, M.; Teker, A. Phys. Rev. B 2014, 89, 144506. doi:10.1103/PhysRevB.89.144506

51. Aasen, D.; Hell, M.; Mishmash, R. V.; Higginbotham, A.; Danon, J.; Leijnse, M.; Jespersen, T. S.; Folk, J. A.; Marcus, C. M.; Flensberg, K.; Alicea, J. Phys. Rev. X 2016, 6, 031016. doi:10.1103/physrevx.6.031016

52. Karzig, T.; Knapp, C.; Lutchyn, R. M.; Bonderson, P.; Hastings, M. B.; Nayak, C.; Alicea, J.; Flensberg, K.; Plugge, S.; Oreg, Y.;

Marcus, C. M.; Freedman, M. H. Phys. Rev. B 2017, 95, 235305. doi:10.1103/PhysRevB.95.235305

53. Sitek, A.; Thorgilsson, G.; Gudmundsson, V.; Manolescu, A. Nanotechnology 2016, 27, 225202. doi:10.1088/0957-4484/27/22/225202

54. Bringer, A.; Schäpers, T. Phys. Rev. B 2011, 83, 115305. doi:10.1103/PhysRevB.83.115305

55. Buscemi, F.; Royo, M.; Bertoni, A.; Goldoni, G. Phys. Rev. B 2015, 92, 165302. doi:10.1103/PhysRevB.92.165302

56. Woods, B. D.; Stanescu, T. D.; Das Sarma, S. arXiv 2018, 1801.02630

57. Sitek, A.; Țolea, M.; Niță, M.; Serra, L.; Gudmundsson, V.; Manolescu, A. Sci. Rep. 2017, 7, 40197. doi:10.1038/srep40197

58. Gangadharaiah, S.; Braunecker, B.; Simon, P.; Loss, D. Phys. Rev. Lett. 2011, 107, 036811. doi:10.1103/PhysRevLett.107.036801 
59. Sela, E.; Altland, A.; Rosch, A. Phys. Rev. B 2011, 84, 085114 doi:10.1103/PhysRevB.84.085114

60. Lutchyn, R. M.; Fisher, M. P. A. Phys. Rev. B 2011, 84, 214528. doi:10.1103/PhysRevB.84.214528

61. Stoudenmire, E. M.; Alicea, J.; Starykh, O. A.; Fisher, M. P. A. Phys. Rev. B 2011, 84, 014503. doi:10.1103/PhysRevB.84.014503

62. Lobos, A. M.; Lutchyn, R. M.; Das Sarma, S. Phys. Rev. Lett. 2012, 109, 146403. doi:10.1103/PhysRevLett.109.146403

63. Hassler, F.; Schuricht, D. New J. Phys. 2012, 14, 125018. doi:10.1088/1367-2630/14/12/125018

64. Thomale, R.; Rachel, S.; Schmitteckert, P. Phys. Rev. B 2013, 88, 161103. doi:10.1103/PhysRevB.88.161103

65. Manolescu, A.; Marinescu, D. C.; Stanescu, T. D. J. Phys.: Condens. Matter 2014, 26, 172203. doi:10.1088/0953-8984/26/17/172203

\section{License and Terms}

This is an Open Access article under the terms of the Creative Commons Attribution License

(http://creativecommons.org/licenses/by/4.0), which permits unrestricted use, distribution, and reproduction in any medium, provided the original work is properly cited.

The license is subject to the Beilstein Journal of

Nanotechnology terms and conditions:

(https://www.beilstein-journals.org/bjnano)

The definitive version of this article is the electronic one which can be found at: doi:10.3762/bjnano.9.142 